\title{
Cosmological gravitational wave background from primordial density perturbations
}

\author{
Kishore N. Ananda, ${ }^{1, *}$ Chris Clarkson, ${ }^{1,2, \dagger}$ and David Wands ${ }^{1, \ddagger}$ \\ ${ }^{1}$ Institute of Cosmology and Gravitation, University of Portsmouth, Mercantile House, Portsmouth PO1 2EG, United Kingdom \\ ${ }^{2}$ Cosmology and Gravity Group, Department of Mathematics and Applied Mathematics, \\ University of Cape Town, Rondebosch 7701, Cape Town, South Africa
}

(Received 13 March 2007; published 26 June 2007)

\begin{abstract}
We discuss the gravitational wave background generated by primordial density perturbations evolving during the radiation era. At second order in a perturbative expansion, density fluctuations produce gravitational waves. We calculate the power spectra of gravitational waves from this mechanism, and show that, in principle, future gravitational wave detectors could be used to constrain the primordial power spectrum on scales vastly different from those currently being probed by a large-scale structure. As examples we compute the gravitational wave background generated by both a power-law spectrum on all scales, and a delta-function power spectrum on a single scale.
\end{abstract}

DOI: 10.1103/PhysRevD.75.123518

PACS numbers: 98.80.Cq, 04.30.Db

\section{INTRODUCTION}

Gravitational waves are an inevitable, yet still elusive, consequence of Einstein's theory of general relativity that will be tested and, we hope, revealed by upcoming experiments. Linear perturbations about a cosmological metric include transverse and trace-free modes (tensor modes) that propagate independently of conventional matter perturbations at first order-i.e., gravitational waves. During an inflationary expansion in the very early universe, largescale (super-Hubble scale) tensor modes will be generated from initial quantum fluctuations on small scales [1-4]. But the amplitude depends on the energy scale of inflation and may be unobservably small if inflation occurs much below the GUT scale [5].

On the other hand, primordial density perturbations and their associated scalar metric perturbations do exist and these will inevitably generate a cosmological background of gravitational waves at second order through mode coupling [6-14]. Given the detailed information we now have about the primordial density perturbations on a range of cosmological scales, it is now timely to consider the amplitude and distribution of tensor (and vector) modes that will be generated at second order.

The observed Gaussian distribution of the primordial density perturbations will generate second-order modes, which will have a $\chi^{2}$-distribution, unlike any first-order gravitational waves from inflation. Given the observed primordial power spectrum of scalar perturbations on large scales, of order $10^{-9}$ [15], one would expect the power spectrum of second-order metric perturbations to be of order $10^{-18}$ in the radiation-dominated era. But this naive expectation needs to be tested against a full second-order calculation. To our knowledge this has not previously been done. Recently the effect on the cosmic microwave back-

\footnotetext{
*kishore.ananda@port.ac.uk

†chris.clarkson@uct.ac.za

‡avid.wands@port.ac.uk
}

ground $(\mathrm{CMB})$ of second-order gravitational waves generated on very large scales was investigated by Mollerach et al. [16] but these modes enter the Hubble scale after matter-radiation equality. We will consider much smaller scale modes that enter the Hubble scale during the primordial radiation-dominated era and may be relevant for direct detection by gravitational wave experiments.

Moreover, the generation of gravitational waves from primordial density perturbations on smaller scales, not directly probed by astronomical observations, could be used in the same way that primordial black hole formation has previously been used to constrain overdensities on these scales [17]. Recently, Easther and Lim [18] (see also Refs. [19-21]) have suggested that large density inhomogeneities on sub-Hubble scales during preheating at the end of inflation could generate a gravitational wave background that might be detectable by future gravitational wave experiments such as Advanced LIGO [22].

In this paper we present the second-order evolution equation for gravitational waves generated from terms quadratic in the first-order matter and metric perturbations. In Sec. II we present the field equations for the background Friedmann-Lemaître-Robertson-Walker (FLRW) metric and first-order perturbations, giving the standard solutions in terms of the Bardeen metric potential during the primordial radiation-dominated era. In Sec. III we present the second-order evolution equation for gravitational waves driven by a source which is quadratic in the first-order scalar perturbations. We use the first-order constraint equations to eliminate the matter perturbations and write this evolution equation solely in terms of the Bardeen potentials and their derivatives. Finally, we present solutions to the second-order gravitational wave equation using a Green function method. We calculate the power spectrum of the gravitational waves generated first by a deltafunction spectrum of density perturbations at a particular wavelength, and then by a power-law spectrum for the primordial density perturbations. We conclude by discussing the expected amplitudes of the gravitational waves 
produced and compare with sensitivities of current and future detectors in Sec. IV.

\section{DENSITY FLUCTUATIONS IN THE RADIATION ERA}

We will consider perturbations up to second order about a Robertson-Walker background. We decompose the metric as [23]

$$
\bar{g}_{\alpha \beta}=g_{\alpha \beta}+\delta g_{\alpha \beta}+\delta^{2} g_{\alpha \beta} .
$$

As we are considering gravitational waves sourced by firstorder scalar perturbations, $\delta g_{\alpha \beta}$ has purely scalar degrees of freedom, while $\delta^{2} g_{\alpha \beta}$ has, in general, scalar, vector, and tensor modes induced by $\delta g_{\alpha \beta}$. However, we are only investigating second-order tensor modes so will project out any scalar or vector modes at second order; we may therefore consider $\delta^{2} g_{\alpha \beta}$ as having purely tensor degrees of freedom. Choosing a longitudinal gauge at first order, we write the metric as

$$
\begin{gathered}
\bar{g}_{00}=-a^{2}[1+2 \Phi], \quad \bar{g}_{0 i}=0, \\
\bar{g}_{i j}=a^{2}\left[(1-2 \Phi) \gamma_{i j}+\frac{1}{2} h_{i j}\right] .
\end{gathered}
$$

Here, $\Phi$ is the first-order Bardeen potential, and $h_{i j}$ is the second-order tensor mode. Note that $\gamma^{j k} h_{i j \mid k}=0$ and $\gamma^{i j} h_{i j}=0$.

We assume a spatially flat geometry and a pure radiation background. The scale factor, Hubble rate, and energy density evolve as $a=a_{0}\left(\frac{\eta}{\eta_{0}}\right), \mathcal{H}=a H=\eta^{-1}$, and $\rho \propto$ $\eta^{-4}$, in terms of conformal time $\eta$ (we fix the conformal time today to be $\eta_{0}=1$ ).

At first order, assuming no anisotropic pressure, the Bardeen potential, for a comoving wave number $k$, satisfies

$$
\Phi^{\prime \prime}+3 \mathcal{H}\left(1+c_{s}^{2}\right) \Phi^{\prime}+c_{s}^{2} k^{2} \Phi=0,
$$

where the speed of sound is $c_{s}^{2}=1 / 3$, and a prime denotes a derivative with respect to conformal time. The general solution to this is

$$
\begin{aligned}
\Phi(\boldsymbol{k}, \boldsymbol{\eta})= & \frac{A(\boldsymbol{k})}{(k \boldsymbol{\eta})^{3}}\left[\frac{k \eta}{\sqrt{3}} \cos \left(\frac{k \eta}{\sqrt{3}}\right)-\sin \left(\frac{k \eta}{\sqrt{3}}\right)\right] \\
& +\frac{B(\boldsymbol{k})}{(k \eta)^{3}}\left[\frac{k \eta}{\sqrt{3}} \sin \left(\frac{k \eta}{\sqrt{3}}\right)+\cos \left(\frac{k \boldsymbol{\eta}}{\sqrt{3}}\right)\right] .
\end{aligned}
$$

This will act as a source for the gravitational waves (GW's) at second order. At early times, $k \eta \rightarrow 0$, we see that

$$
\Phi(\boldsymbol{k}, \eta)=-\frac{A(\boldsymbol{k})}{9 \sqrt{3}}+\frac{B(\boldsymbol{k})}{(k \eta)^{3}}
$$

the second term is the decaying mode which we shall neglect hereafter.

Assuming that the fluctuations are Gaussian, we may write $A(\boldsymbol{k})=A(k) \hat{E}(\boldsymbol{k})$, where the $\hat{E}$ are Gaussian random variables of unit variance which have the property

$$
\left\langle\hat{E}^{*}\left(\boldsymbol{k}_{1}\right) \hat{E}\left(\boldsymbol{k}_{2}\right)\right\rangle=\delta^{3}\left(\boldsymbol{k}_{1}-\boldsymbol{k}_{2}\right) .
$$

The power spectrum for the scalar perturbation can then be defined as

$$
\left\langle\Phi^{*}\left(\boldsymbol{k}_{1}\right) \Phi\left(\boldsymbol{k}_{2}\right)\right\rangle=\frac{2 \pi^{2}}{k^{3}} \delta\left(\boldsymbol{k}_{1}-\boldsymbol{k}_{2}\right) \mathcal{P}_{\Phi}(k, \eta),
$$

implying that at early times the power spectrum becomes

$$
\mathcal{P}_{\Phi}(k) \simeq A(k)^{2} \frac{k^{3}}{486 \pi^{2}} .
$$

The Bardeen potential can be related to the comoving curvature perturbation at early times, giving

$$
A(k)^{2} \approx \frac{216 \pi^{2}}{k^{3}} \Delta_{\mathcal{R}}^{2}(k),
$$

where $\Delta_{\mathcal{R}}^{2}$ is the primordial power spectrum for the curvature perturbation $\mathcal{R}$. Current observations show $\Delta_{\mathcal{R}}^{2} \simeq 2 \times$ $10^{-9}$ at a scale $k_{\mathrm{CMB}}=0.002 \mathrm{Mpc}^{-1}$, and is almost independent of wave number on these scales [15].

\section{THE INDUCED GRAVITATIONAL WAVES}

We now consider the evolution equations for the secondorder tensor perturbations, $h_{i j}$, sourced by the scalar density perturbations discussed above.

We will write the Fourier transform of $h_{i j}$ as

$$
\begin{aligned}
h_{i j}(\boldsymbol{x}, \eta)= & \frac{1}{(2 \pi)^{3 / 2}} \int \mathrm{d}^{3} k e^{i \boldsymbol{k} \cdot \boldsymbol{x}}\left[h(\boldsymbol{k}, \eta) q_{i j}(\boldsymbol{k})\right. \\
& \left.+\bar{h}(\boldsymbol{k}, \eta) \bar{q}_{i j}(\boldsymbol{k})\right]
\end{aligned}
$$

where the two polarization tensors $q_{i j}$ and $\bar{q}_{i j}$ are expressed in terms of the orthonormal basis vectors $\boldsymbol{e}$ and $\overline{\boldsymbol{e}}$ orthogonal to $\boldsymbol{k}$,

$$
\begin{aligned}
& q_{i j}(\boldsymbol{k})=\frac{1}{\sqrt{2}}\left[e_{i}(\boldsymbol{k}) e_{j}(\boldsymbol{k})-\bar{e}_{i}(\boldsymbol{k}) \bar{e}_{j}(\boldsymbol{k})\right], \\
& \bar{q}_{i j}(\boldsymbol{k})=\frac{1}{\sqrt{2}}\left[e_{i}(\boldsymbol{k}) \bar{e}_{j}(\boldsymbol{k})+\bar{e}_{i}(\boldsymbol{k}) e_{j}(\boldsymbol{k})\right] .
\end{aligned}
$$

Thus to extract the transverse, trace-free part of any tensor we project with the operator $\hat{\mathcal{T}}_{i j}{ }^{l m}$, defined through its action on a two-index tensor

$$
\begin{aligned}
\hat{\mathcal{T}}_{i j}^{l m} \mathcal{S}_{l m}= & \int d^{3} k^{\prime} \frac{q_{i j}\left(\boldsymbol{k}^{\prime}\right)}{(2 \pi)^{3 / 2}} \\
& \times \int d^{3} x^{\prime} \frac{q^{l m}\left(\boldsymbol{k}^{\prime}\right)}{(2 \pi)^{3 / 2}} e^{i \boldsymbol{k}^{\prime} \cdot\left(\boldsymbol{x}-\boldsymbol{x}^{\prime}\right)} \mathcal{S}_{l m}\left(\boldsymbol{x}^{\prime}\right) \\
& +\int d^{3} k^{\prime} \frac{\bar{q}_{i j}\left(\boldsymbol{k}^{\prime}\right)}{(2 \pi)^{3 / 2}} \\
& \times \int d^{3} x^{\prime} \frac{\bar{q}^{l m}\left(\boldsymbol{k}^{\prime}\right)}{(2 \pi)^{3 / 2}} e^{i \boldsymbol{k}^{\prime} \cdot\left(\boldsymbol{x}-\boldsymbol{x}^{\prime}\right)} \mathcal{S}_{l m}\left(\boldsymbol{x}^{\prime}\right) .
\end{aligned}
$$


The evolution equation for the second-order tensors is calculated by expanding the Einstein field equation to second order. The Ricci tensor includes terms linear in the second-order tensor perturbations and quadratic terms in the first-order scalar perturbations $[9,24]$. This is equated with second-order terms in the trace-reversed energymomentum tensor. The only contributions from pure second-order matter fluctuations would come from a transverse and trace-free contribution to the anisotropic stress which we ignore in this analysis. On the other hand, there are terms quadratic in the scalar density and velocity perturbations, which can be written in terms of the scalar metric perturbations using the first-order Einstein constraint equations (the relevant terms can be found in Appendix A). Finally, we act on both the Ricci tensor and the trace-reversed energy-momentum tensor with the operator $\hat{\mathcal{T}}_{i j}{ }^{l m}$, defined above, in order to pick out the transverse and trace-free parts of the evolution equation. This yields $[25,26]$

$$
h_{i j}^{\prime \prime}+2 \mathcal{H} h_{i j}^{\prime}-\nabla^{2} h_{i j}=-4 \hat{\mathcal{T}}_{i j}{ }^{l m} \mathcal{S}_{l m} .
$$

The source term is given by $[25,26]$

$$
\begin{aligned}
\mathcal{S}_{i j}= & 4 \Phi \Phi_{\mid i j}+2 \Phi_{\mid i} \Phi_{\mid j}-\frac{3}{\kappa^{2} a^{2} \rho}\left[\mathcal{H}^{2} \Phi_{\mid i} \Phi_{\mid j}\right. \\
& \left.+2 \mathcal{H} \Phi_{\mid i} \Phi_{\mid j}^{\prime}+\Phi_{\mid i}^{\prime} \Phi_{\mid j}^{\prime}\right] .
\end{aligned}
$$

A pipe denotes the spatial covariant derivative, and $\kappa^{2}=$ $8 \pi G$. This expression is consistent with the second-order Ricci tensor for scalar perturbations about an FLRW background calculated in Ref. [24].

In Fourier space we then find the amplitude of the tensor mode, for either polarization, obeys the evolution equation

$$
h^{\prime \prime}(\boldsymbol{k}, \eta)+\frac{2}{\eta} h^{\prime}(\boldsymbol{k}, \eta)+k^{2} h(\boldsymbol{k}, \eta)=\mathcal{S}(\boldsymbol{k}, \eta),
$$

where the source term is given by

$$
\begin{aligned}
\mathcal{S}(\boldsymbol{k}, \tilde{\eta})= & \frac{q^{i j}(\boldsymbol{k})}{(2 \pi)^{3 / 2}} \int d^{3} \tilde{k} \tilde{k}_{i} \tilde{k}_{j}\{12 \Phi(\boldsymbol{k}-\tilde{\boldsymbol{k}}, \tilde{\eta}) \Phi(\tilde{\boldsymbol{k}}, \tilde{\eta}) \\
& +\left[\tilde{\eta} \Phi(\boldsymbol{k}-\tilde{\boldsymbol{k}}, \tilde{\eta})+\frac{\tilde{\eta}^{2}}{2} \Phi^{\prime}(\boldsymbol{k}-\tilde{\boldsymbol{k}}, \tilde{\eta})\right] \\
& \left.\times \Phi^{\prime}(\tilde{\boldsymbol{k}}, \tilde{\eta})\right\} .
\end{aligned}
$$

The particular solution for the gravitational waves is then given by an integral over the Green's function

$$
h(\boldsymbol{k}, \eta)=\frac{1}{a(\eta)} \int_{\eta_{0}}^{\eta} G_{k}(\eta, \tilde{\eta}) a(\tilde{\eta}) \mathcal{S}(\boldsymbol{k}, \tilde{\eta}) d \tilde{\eta},
$$

where

$$
G_{k}(\eta, \tilde{\eta})=\frac{4}{\pi^{2} k}[\sin (k \eta) \cos (k \tilde{\eta})-\cos (k \eta) \sin (k \tilde{\eta})] .
$$

We have imposed the initial conditions, $h\left(\boldsymbol{k}, \eta_{0}\right)=0$ and $h^{\prime}\left(\boldsymbol{k}, \eta_{0}\right)=0$ which is consistent with the assumption that there are no GW's from inflation. It should be noted that, if first-order tensor modes were present, they would be uncorrelated with the first-order scalar modes and hence uncorrelated with the induced second-order tensor modes.

The power spectrum of the induced GW is defined in the usual manner,

$$
\left\langle h\left(\boldsymbol{k}_{1}, \eta\right) h\left(\boldsymbol{k}_{2}, \eta\right)\right\rangle=\frac{2 \pi^{2}}{k^{3}} \delta\left(\boldsymbol{k}_{1}-\boldsymbol{k}_{2}\right) \mathcal{P}_{h}(k, \eta) .
$$

Substituting for $h(\boldsymbol{k}, \eta)$ and using Wick's theorem, and using spherical coordinates in Fourier space we find that

$$
\begin{aligned}
\mathcal{P}_{h}(k, \eta)= & \frac{1}{8 \pi^{4} a(\eta)^{2}} \int_{\eta_{0}}^{\eta} d \tilde{\eta}_{2} \int_{\eta_{0}}^{\eta} d \tilde{\eta}_{1} a\left(\tilde{\eta}_{1}\right) a\left(\tilde{\eta}_{2}\right) \\
& \times \int_{0}^{\infty} d \tilde{k} \int_{u_{-}}^{u_{+}} d u k^{2} G_{k}\left(\eta, \tilde{\eta}_{1}\right) G_{k}\left(\eta, \tilde{\eta}_{2}\right) \\
& \times F\left(\tilde{k}, k, u ; \tilde{\eta}_{1}, \tilde{\eta}_{2}\right) .
\end{aligned}
$$

The variable $u$ is given by

$$
u=\sqrt{1+(\tilde{k} / k)^{2}-2(\tilde{k} / k) \cos \theta},
$$

where $\theta$ is the angle between the modes $\boldsymbol{k}$ and $\tilde{\boldsymbol{k}}$, and the limits of integration $u_{ \pm}$correspond to the angles $\theta=0, \pi$, respectively. The integrand $F$ in Eq. (20) is found to be

$$
\begin{aligned}
F\left(\tilde{k}, k, u ; \tilde{\eta}_{1}, \tilde{\eta}_{2}\right)= & u \tilde{k}\left[(2 k \tilde{k})^{2}-\left((u k)^{2}-k^{2}-\tilde{k}^{2}\right)^{2}\right]^{2}\left\{3 \Phi\left(u k, \tilde{\eta}_{1}\right) \Phi\left(\tilde{k}, \tilde{\eta}_{1}\right)+\left[2 \tilde{\eta}_{1} \Phi\left(u k, \tilde{\eta}_{1}\right)+\tilde{\eta}_{1}^{2} \Phi^{\prime}\left(u k, \tilde{\eta}_{1}\right)\right] \Phi^{\prime}\left(\tilde{k}, \tilde{\eta}_{1}\right)\right\} \\
& \times\left\{3 \Phi\left(u k, \tilde{\eta}_{2}\right) \Phi\left(\tilde{k}, \tilde{\eta}_{2}\right)+\tilde{\eta}_{2}^{2} \Phi^{\prime}\left(u k, \tilde{\eta}_{2}\right) \Phi^{\prime}\left(\tilde{k}, \tilde{\eta}_{2}\right)+\tilde{\eta}_{2}\left[\Phi\left(u k, \tilde{\eta}_{2}\right) \Phi^{\prime}\left(\tilde{k}, \tilde{\eta}_{2}\right)+\Phi\left(\tilde{k}, \tilde{\eta}_{2}\right) \Phi^{\prime}\left(u k, \tilde{\eta}_{2}\right)\right]\right\},
\end{aligned}
$$

where we distinguish between the Gaussian random variable $\Phi(\boldsymbol{k}, \eta)$ in Eq. (16) and its amplitude $\Phi(k, \eta)$ in Eq. (22) which we take to be isotropic. We introduce the following dimensionless variables: 


$$
v=\frac{\tilde{k}}{k}, \quad x=k \eta
$$

and substitute for the first-order solution for $\Phi$ from Eq. (4), as well as the Green's function, into our GW power spectrum. After some simplification and substitution from the above formulas we have

$$
\begin{aligned}
\mathcal{P}_{h}(k, \eta)= & \frac{2(216)^{2}}{\pi^{4} \eta^{2}} \int_{0}^{\infty} d v \int_{|v-1|}^{|v+1|} d u \frac{1}{(u v)^{8}} \\
& \times\left[4 v^{2}-\left(u^{2}-v^{2}-1\right)^{2}\right]^{2} \mathcal{P}_{\Phi}(u k) \mathcal{P}_{\Phi}(v k) \\
& \times\left[\sin (x) \int_{x_{0}}^{x} d \tilde{x}_{1} I_{1}\left(\tilde{x}_{1}\right)-\cos (x) \int_{x_{0}}^{x} d \tilde{x}_{1} I_{2}\left(\tilde{x}_{1}\right)\right] \\
& \times\left[\sin (x) \int_{x_{0}}^{x} d \tilde{x}_{2} I_{3}\left(\tilde{x}_{2}\right)-\cos (x) \int_{x_{0}}^{x} d \tilde{x}_{2} I_{4}\left(\tilde{x}_{2}\right)\right],
\end{aligned}
$$

where we have defined the four functions

$$
I_{j}(x)=\sum_{m=1}^{5} \sum_{n=1}^{8} \sin \left(\alpha_{n} x+\phi_{n}\right) \frac{M_{n m}^{j}}{x^{m}} .
$$

The coefficients $\alpha_{n}, \phi_{n}$, and $M_{n m}^{j}$ in this expression are dependent on $u$ and $v$ but not $x$, and may be found in Appendix B.

Evaluating the integrals in the right-hand side of Eq. (24) for various input scalar power spectra will then tell us the power in each GW mode. This is not particularly simple, so we start by analytically expanding the integrals over the functions $I_{j}$ by parts up to $\mathrm{Si}$ and $\mathrm{Ci}$ functions [27]:

$$
\begin{aligned}
X_{j}\left(u, v, x, x_{0}\right)= & \int_{x_{0}}^{x} d \tilde{x} I_{j}(\tilde{x}) \\
= & \sum_{m=1}^{5} \sum_{n=1}^{8} M_{n m}^{j}\left\{\left[\sum_{k=1}^{m-2} \frac{(m-k-2) !}{(m-1) !} \alpha_{n}^{k}\right.\right. \\
& \left.\times \sin \left(\alpha_{n} \tilde{x}+\phi_{n}+\frac{(k+2)}{2} \pi\right) \tilde{x}^{(1+k-m)}\right]_{x_{0}}^{x} \\
& -\frac{\alpha_{n}^{(m-1)}}{(m-1) !} \int_{x_{0}}^{x} d \tilde{x} \frac{1}{\tilde{x}} \\
& \left.\times \sin \left(\alpha_{n} \tilde{x}+\phi_{n}+\frac{(m+1)}{2} \pi\right)\right\} .
\end{aligned}
$$

The remaining two integrals over Fourier space can now be done numerically once power spectra for the scalar modes are chosen. We shall only consider modes which start their evolution well outside the Hubble radius, and hereafter set $x_{0}=0$.

\section{A. Gravitational wave generation by a single scalar mode}

Second-order gravitational waves potentially provide a method by which we could detect a particular scalar mode with excessive power, compared to the roughly scale- invariant average we observe on large scales today. As a precursor to a power-law power spectrum for the scalar modes, we can investigate how the system reacts to power being put in at one particular scale. That is, we put power in at a single wavelength (i.e., a single comoving scale) which is described by an isotropic distribution of wave vectors (i.e., at all possible angles). This would be useful when considering preheating, for example, which can result in features (large power over narrow range of scales) in the power spectrum of the input scalar perturbations $[18,19]$.

We will therefore consider the case of the delta-function power spectrum, as an idealized limit of a spike in the power spectrum, or of power being introduced on a narrow range of scales above the roughly scale-invariant primordial spectrum observed. The specific form we choose is

$$
\mathcal{P}_{\Phi}(k)=\frac{4}{9} \mathcal{A}^{2} \Delta_{\mathcal{R}}^{2}\left(k_{\mathrm{CMB}}\right) \delta\left(\ln \left(k / k_{\mathrm{in}}\right)\right),
$$

where $\mathcal{A}$ is the amplitude at a single wave number, $k_{\text {in }}$, relative to the observed amplitude of the primordial power spectrum, $\Delta_{\mathcal{R}}^{2}\left(k_{\mathrm{CMB}}\right)$, at wave number $k_{\mathrm{CMB}} \gg k_{\text {in }}$.

The power spectrum of the gravitational waves produced by this scalar mode is then, from Eq. (24),

$$
\begin{aligned}
\mathcal{P}_{h}(k, \eta)= & \frac{32(216)^{2}}{81 \pi^{4} \eta^{2}} \int_{0}^{\infty} d v^{\prime} \int_{|v-1|}^{|v+1|} d u^{\prime} \mathcal{P}_{\Phi}\left(u^{\prime} k\right) \\
& \times \mathcal{P}_{\Phi}\left(v^{\prime} k\right) \mathcal{F}_{\delta}\left(u^{\prime}, v^{\prime}, x\right),
\end{aligned}
$$

where

$$
\begin{aligned}
\mathcal{F}_{\delta}(u, v, x)= & \frac{1}{(u v)^{8}}\left[4 v^{2}-\left(u^{2}-v^{2}-1\right)^{2}\right]^{2}\left[\sin (x) X_{1}\right. \\
& \left.-\cos (x) X_{2}\right]\left[\sin (x) X_{3}-\cos (x) X_{4}\right] .
\end{aligned}
$$

We may now evaluate the $u^{\prime}$ and $v^{\prime}$ integrals for a deltafunction power spectrum, giving

$$
\mathcal{P}_{h}(k, \eta)=\frac{32(216)^{2}}{81 \pi^{4} \eta^{2}} \mathcal{A}^{4} \Delta_{\mathcal{R}}^{4}\left(\frac{k_{\text {in }}}{k}\right)^{2} \mathcal{F}_{\delta}(u, v, x),
$$

provided that the following condition holds:

$$
u=v=\frac{k_{\text {in }}}{k} \geq \frac{1}{2} .
$$

The inequality arises from the fact that gravitational waves cannot be excited with more than twice the momentum of the density perturbations, $k_{\text {in }}$.

Although $\mathcal{F}_{\delta}(v, v, x)$ is a very complicated function to write down, its properties are relatively straightforward to understand, as we can see from Figs. 1-4.

In Fig. 1 we see the basic behavior for small $x$. When $x_{0}=0$, we may interpret $x$ as time for a fixed wavelength or wave number at fixed time. The function therefore is zero at early times and large scales, has interesting oscillatory behavior on scales of order the horizon size, when $k \eta=1$, and oscillates at constant amplitude at small scales and/or late times - i.e., when modes are well inside the horizon. The asymptotic behavior is clearly seen in 


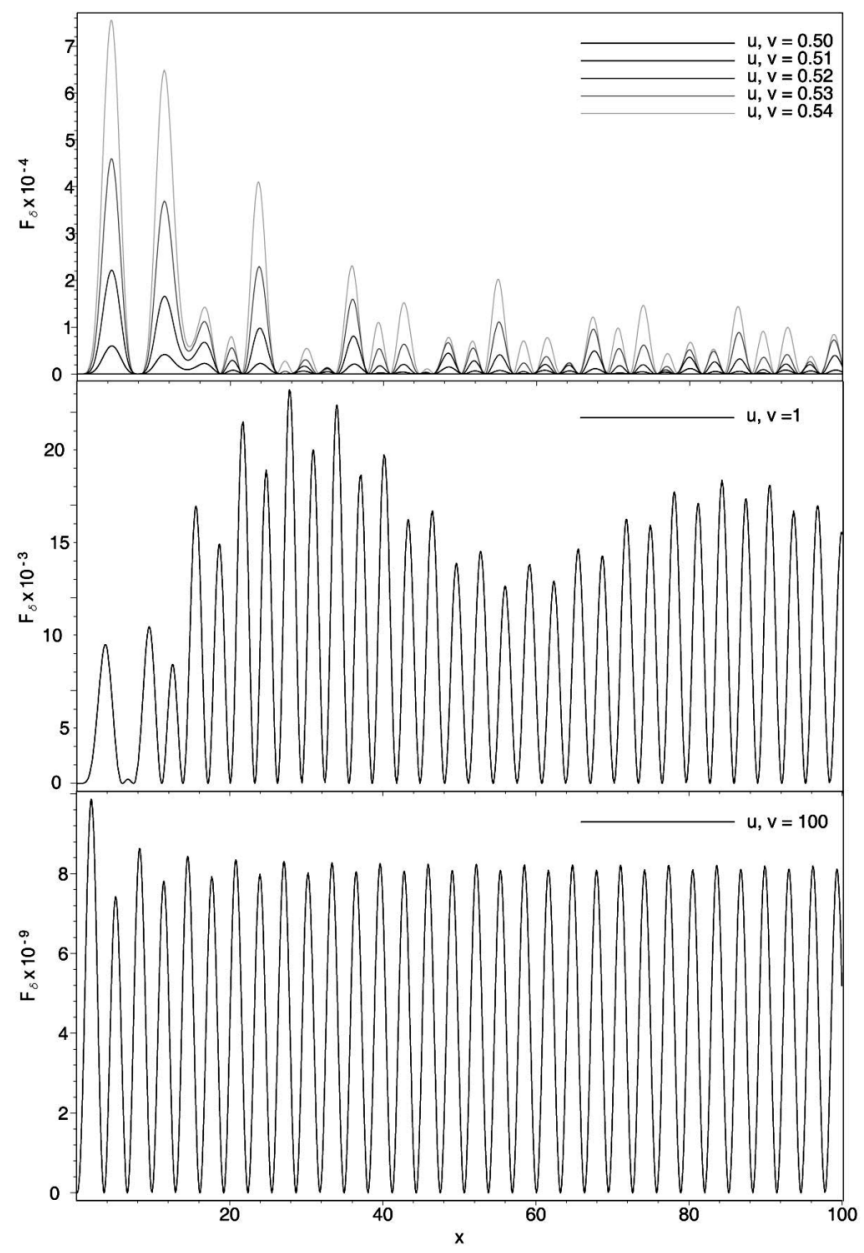

FIG. 1. The function $\mathcal{F}_{\delta}(x)$ for different values of $k_{\text {in }} / k=$ $u=v$, with $x_{0}=0$.

Fig. 2, where we are looking at modes with $k=k_{\text {in }}$, generated when there is a 60 degree angle $\left[\theta=60^{\circ}\right.$ ) between the input and output mode (where $\theta$ is given by Eqs. (21) and (23)]. We have power-law growth for small $x$, and constant amplitude for $x \rightarrow \infty$, with interesting oscillations on scales of the order of the Hubble scale. In Fig. 3 we show the same but now with $u=v=\sqrt{3} / 2$. We see that the amplitude continues to grow on small scales or late times, corresponding to resonant amplification of modes generated at an angle $\cos \theta=1 / \sqrt{3}$ to the incoming mode.

In Fig. 4 we show the envelope of $\mathcal{F}_{\delta}$ at fixed large $x$ (where we have chosen $x=10^{20}$ ) which shows the shape of the power spectrum one could observe at late times. There is a power-law tail on large scales, $k \ll k_{\text {in }}$, which is proportional to $\left(k / k_{\text {in }}\right)^{3}$ as one would expect for perturbations generated from much smaller scale modes and hence uncorrelated on larger scales. On small scales there is a sharp high-frequency cutoff at $k=2 k_{\text {in }}$. We also see the resonance as a sharp spike in the power spectrum, of approximate width $1 / x$ and amplitude $0.05\left(\log _{10} x\right) \times$ $\left(\log _{10} 0.02 x\right)$. Finally, we note there is zero power at $u=$

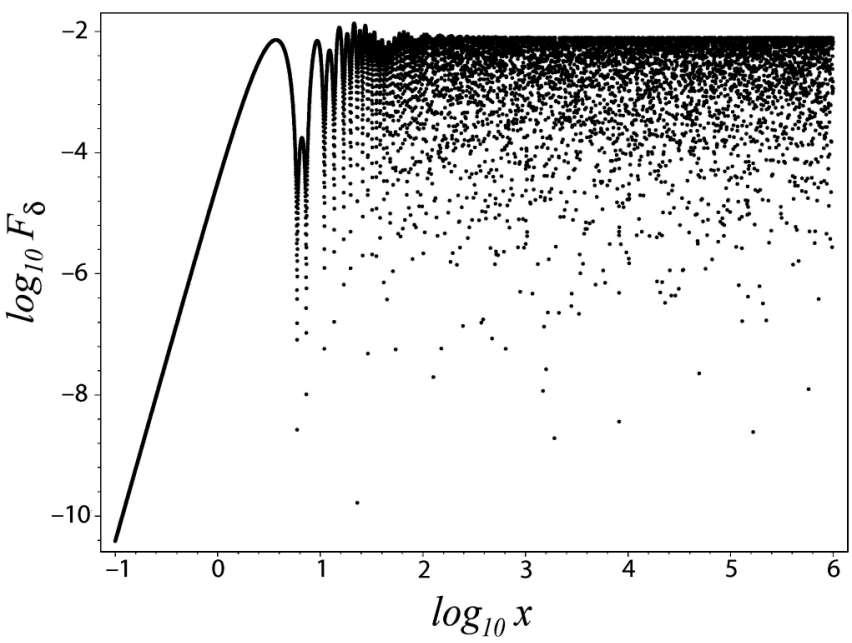

FIG. 2. The function $\mathcal{F}_{\delta}(x)$ from early times (super-Hubble scales) to late times (small scales) for $k=k_{\text {in }}$ (i.e. $u=v=1$ ), $x_{0}=0$. For large $x$ the function oscillates with a constant amplitude.

$v=\sqrt{3 / 2}$, corresponding to an angle $\cos \theta=1 / \sqrt{6}$ between the input and generated modes.

\section{B. Power-law scalar modes}

While the preceding subsection gave insight into many aspects of the generation mechanism, we would also like to know the GW generated from a nearly scale-invariant spectrum of density fluctuations. To investigate this, we assume that the input power spectrum is

$$
\mathcal{P}_{\Phi}\left(k_{\text {in }}\right)=\frac{4}{9} \Delta_{\mathcal{R}}^{2}\left(\frac{k_{\text {in }}}{k_{\mathrm{CMB}}}\right)^{n_{s}-1},
$$

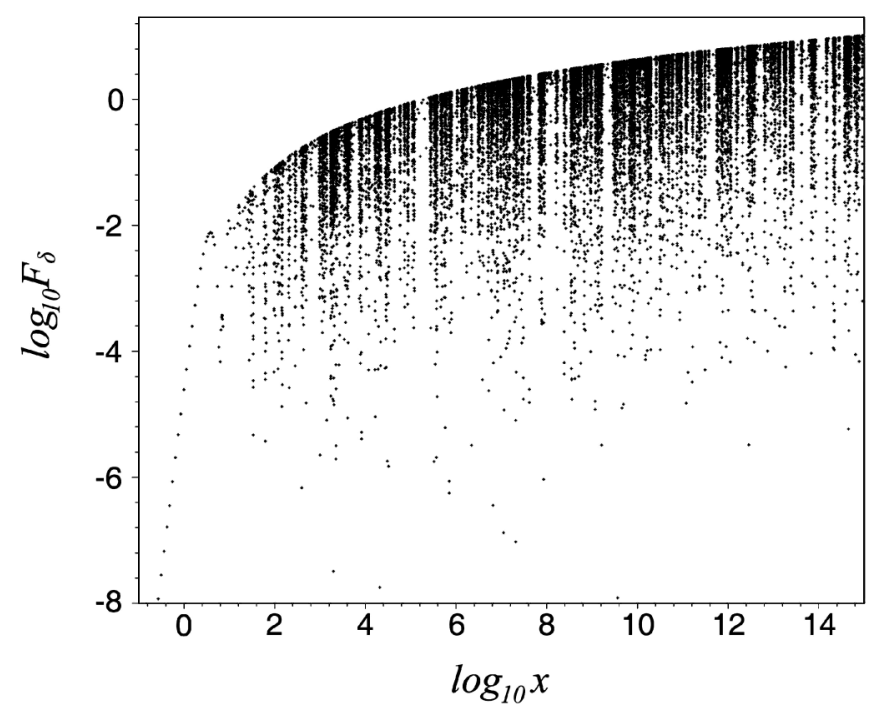

FIG. 3. The function $\mathcal{F}_{\delta}(x)$ from early times (super-Hubble scales) to late times (small scales), for the resonant case of $k_{\text {in }} / k=u=v=\sqrt{3} / 2$. For large $x$ the power in the generated GW continues to grow logarithmically. 


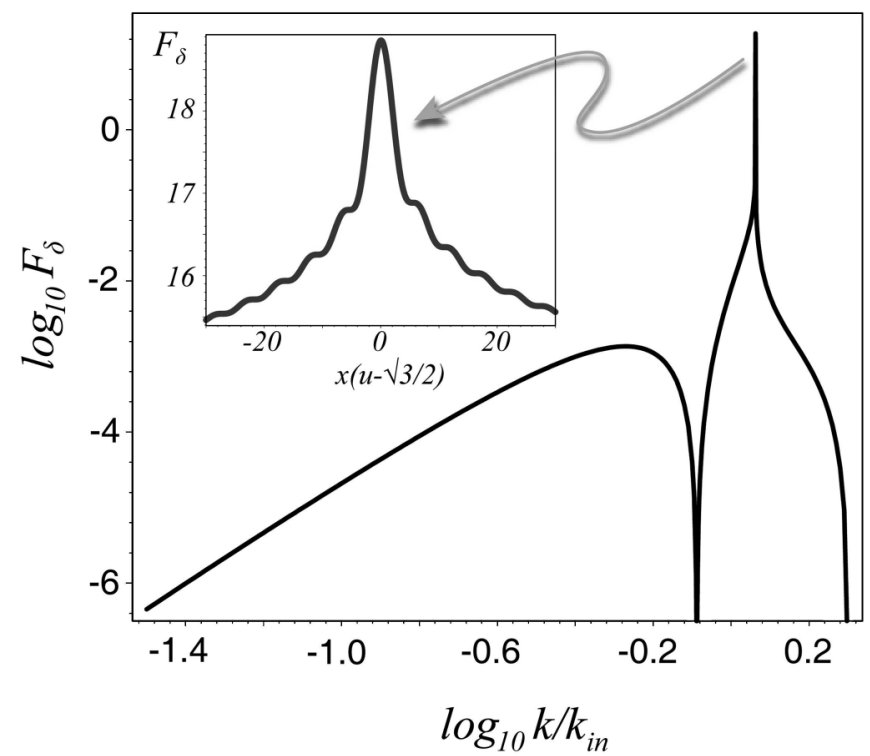

FIG. 4. The power at late times, $x=10^{20}$, for all scales, showing a resonance at $u=\sqrt{3} / 2$ and a power-law tail (due to modes on larger scales being uncorrelated). Detail of the resonance is shown in the inset revealing a wobbly structure.

where the index $n_{s}$ tells us the tilt of the spectrum relative to scale invariance, $n_{s}=1$, and $k_{\mathrm{CMB}}$ is a pivot scale for the power spectrum [15]. The power spectrum of the generated gravitational waves is then given, from Eq. (28), by

$$
\mathcal{P}_{h}(k, \eta)=\frac{32(216)^{2} \Delta_{\mathcal{R}}^{4}}{81 \pi^{4} \eta^{2}}\left(\frac{k}{k_{\mathrm{CMB}}}\right)^{2\left(n_{s}-1\right)} \mathcal{F}_{n_{s}}(x)
$$

The function $\mathcal{F}_{n_{s}}$ is defined by

$$
\mathcal{F}_{n_{s}}(x)=\int_{0}^{\infty} d v \int_{|v-1|}^{|v+1|} d u(u v)^{n_{s}-1} \mathcal{F}_{\delta}(u, v, x) .
$$

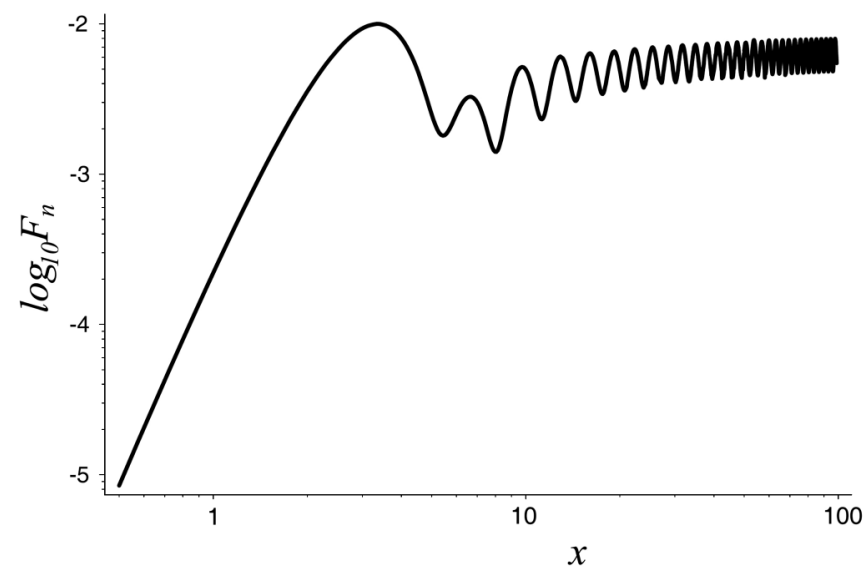

FIG. 5. $\mathcal{F}_{n_{s}}(x)$ for a scale-invariant input power spectrum. For this case the power spectrum of gravitational waves is also scale invariant owing to the scale-invariant conversion factor between $\mathcal{F}_{n_{s}}$ and $\mathcal{P}_{h}$ given by Eq. (33).

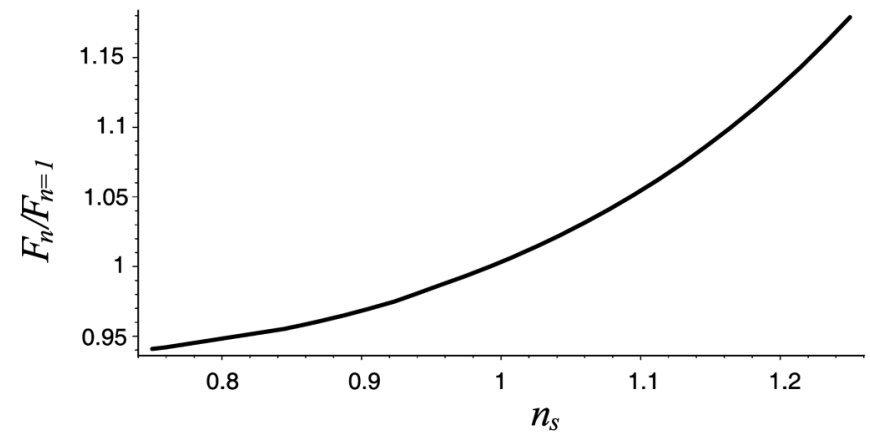

FIG. 6. $\mathcal{F}_{n_{s}}$ for $x \rightarrow \infty, x_{0}=0$, vs the tilt $n_{s}$ of the scalar power spectrum. We have normalized the vertical scale to $\mathcal{F}_{n_{s}=1}=8.3 \times 10^{-3}$.

We show a plot of this function for a scale-invariant spectrum, $n_{s}=1$, in Fig. 5 .

We can think of $\mathcal{F}_{n_{s}}(x)$ as a function of wave number $k=x / \eta$ at a specific time $\eta$, in which case the amplitude of $\mathcal{F}_{n_{s}}$ peaks on scales just inside the Hubble radius, and becomes scale invariant on smaller scales. The series of oscillations for $x>1$ is analogous to the acoustic peaks in the CMB spectrum, although one might have expected this to be less pronounced at second order where there is an integration over modes. It suggests that a narrow range of 1st-order modes with well-defined phase makes the dominant contribution to the second-order tensors on a given scale.

On the other hand, Fig. 5 also represents the evolution of a single mode over time. Thus, power is continually added to the GW until it is well inside the Hubble radius, before oscillating at almost constant amplitude at late times, i.e., a freely propagating $\mathrm{GW}$.

How does the tilt of the scalar modes affect the generated GW? As far as $\mathcal{F}_{n_{s}}$ is concerned there is only a small amplification change, shown in Fig. 6 for large $x$. There is also an increase in the power at small scales due to the factor $\left(k / k_{\mathrm{CMB}}\right)^{2\left(n_{s}-1\right)}$ in Eq. (33) if we consider a blue spectrum $\left(n_{s}>1\right)$.

Finally, we note that for nonzero $x_{0}$ there will be a downward break for large $k$ in the scale invariant $F_{n_{s}}$, which corresponds to $k \eta_{0} \sim 1-$ i.e., modes which are inside the horizon at the start of the interaction period. However, if we start our integration at the GUT scale this will only be relevant for very high frequencies $(~ \gtrsim$ $10^{8} \mathrm{~Hz}$ ), so we shall not pursue this further here.

\section{DISCUSSION}

We have calculated the stochastic background of gravitational waves predicted at second order due to primordial density perturbations and scalar metric perturbations at first order in the early (radiation-dominated) universe. In particular, we have evaluated the power spectrum of gravitational waves produced first, by a delta-function power 
spectrum, representing an infinitely sharp feature in the primordial spectrum at a single wavelength, and secondly by a power-law spectrum, compatible with direct observations of the primordial density perturbations on much larger $(\mathrm{CMB})$ scales. It is interesting to compare these predictions with the sensitivities of current or planned gravitational wave experiments.

The resulting power spectrum of gravitational waves at matter-radiation equality, $\eta_{\text {eq }}$, can be related to their energy density (per logarithmic interval) today by [28]

$$
\Omega_{\mathrm{GW}}(k, \eta)=\frac{\Omega_{\gamma}(\eta)}{\Omega_{\gamma}\left(\eta_{\mathrm{eq}}\right)} \frac{4 \pi}{3} \eta_{\mathrm{eq}}^{2} \mathcal{P}_{h}\left(k, \eta_{\mathrm{eq}}\right)
$$

using the fact that the energy densities of GW and radiation evolve at the same rate in the matter era. We shall consider first the case of the power-law spectrum of primordial density perturbations, for which we obtain

$$
\begin{aligned}
\Omega_{\mathrm{GW}}(k, \eta)= & \frac{\Omega_{\gamma}(\eta)}{\Omega_{\gamma}\left(\eta_{\mathrm{eq}}\right)} \\
& \times \frac{124416}{\pi^{3}} \Delta_{\mathcal{R}}^{4}\left(\frac{k}{k_{\mathrm{CMB}}}\right)^{2\left(n_{s}-1\right)} \mathcal{F}_{n_{s}}\left(k \eta_{\mathrm{eq}}\right) .
\end{aligned}
$$

We have assumed that all modes are outside the Hubble radius at the start of their evolution, $x_{0} \ll 1$.

The latest WMAP data gives $\Delta_{\mathcal{R}}^{2}\left(k_{\mathrm{CMB}}=\right.$ $\left.0.002 \mathrm{Mpc}^{-1}\right) \approx 2.36 \times 10^{-9}$. Combining this with $\Omega_{\gamma} \approx$ $5 \times 10^{-5}$ today, we have

$$
\Omega_{\mathrm{GW}}(k, \eta) \approx 2.23 \times 10^{-18}\left(\frac{k}{k_{\mathrm{CMB}}}\right)^{2\left(n_{s}-1\right)} \mathcal{F}_{n_{s}}\left(k \eta_{\mathrm{eq}}\right) .
$$

The mode which enters the Hubble radius at matterradiation equality defines $\eta_{\text {eq }}=1 / k_{\text {eq }}$, where $k_{\text {eq }}=$ $0.009 \mathrm{Mpc}^{-1}$, corresponding to a GW frequency today of $f_{\text {eq }}=c k_{\text {eq }} / 2 \pi \approx 1.4 \times 10^{-17} \mathrm{~Hz}$. Our pivot value corresponds to frequency $f_{\mathrm{CMB}} \approx 3.1 \times 10^{-18} \mathrm{~Hz}$. For the power-law case we then have

$$
\Omega_{\mathrm{GW}} \approx 1.86 \times 10^{-20+34\left(n_{s}-1\right)}\left(3.2 \frac{f}{\mathrm{~Hz}}\right)^{2\left(n_{s}-1\right)}\left(\frac{\mathcal{F}_{n_{s}}}{\mathcal{F}_{n_{s}=1}}\right),
$$

where $\mathcal{F}_{n_{\mathrm{s}}}$ is evaluated at $x=\infty$, and may be estimated from Fig. 6. Therefore, for a red spectrum with $n_{s}=0.95$, we find $\Omega_{\mathrm{GW}} \approx 3.2 \times 10^{-22}(\mathrm{f} / \mathrm{Hz})^{-0.1}$, while for a blue spectrum with $n_{s}=1.1$ we have $\Omega_{\mathrm{GW}} \approx 6.1 \times$ $10^{-17}(\mathrm{f} / \mathrm{Hz})^{0.2}$. In principle, therefore, proposed detectors such as LISA [29] or DECIGO [30,31] could be used to place limits on the scalar tilt, independently of other observations. Of course, we have only considered constant tilt here; running of the spectral index will change these predictions considerably. Indeed, as the primordial power spectrum is directly constrained only at very large scales,
GWs provide a novel way to probe the primordial power spectrum at wavelengths some 20 orders of magnitude smaller than $\mathrm{CMB}$ measurements allow.

For the case of excess power in a single mode, we may define $\Omega_{\mathrm{GW}}$ analogously with Eq. (35), but near the spike $\mathcal{P}_{h}$ is not smooth, so $\Omega_{\mathrm{GW}}$ is not necessarily the actual energy density of GW. However, we will use it to give a rough indication of power for comparison against quoted detector sensitivities. The peak of the spike in the power spectrum occurs at $f_{\text {peak }}=2 f_{\text {in }} / \sqrt{3}$, which gives

$$
\Omega_{\mathrm{GW}}\left(f_{\text {peak }}\right) \approx 4.5 \times 10^{-17} \mathcal{A}^{4}\left(1+0.09 \log _{10} \frac{T_{\mathrm{ent}}}{1 \mathrm{GeV}}\right) .
$$

We have written this in terms of the temperature at which the input wave number enters the Hubble radius, using [32] $f_{\text {in }}=c k_{\text {in }} / 2 \pi \approx 10^{-6}\left(T_{\text {ent }} / 1 \mathrm{GeV}\right) \mathrm{Hz}$ for $f \gtrsim 10^{-6} \mathrm{~Hz}$, which is the range for any detector of GW. We note that the typical resolution of a detector in time $T$ is $\Delta f \sim 1 / T$ [28]; so, for a one year observation we have $\Delta f \sim 10^{-8} \mathrm{~Hz}$. For current detectors such as GEO [33], LIGO [22], TAMA [34], or VIRGO [35] whose optimal frequency is $\sim 100 \mathrm{~Hz}$, excess power at a single wave number in the primordial power spectrum would correspond to a horizon entry temperature of $T_{\text {ent }} \approx 10^{8} \mathrm{GeV}$, implying that $\Omega_{\mathrm{GW}} \sim 10^{-16} \mathcal{A}^{4}$. While this is nominally 7 orders of magnitude below Advanced LIGO's sensitivity, $\Omega_{\mathrm{GW}} \sim$ $10^{-9}$, it implies that Advanced LIGO could, in principle, pick up modes with $\mathcal{A} \sim 100$, i.e., one hundred times the amplitude observed on large scales. For detectors such as $\mathrm{BBO} / \mathrm{DECIGO}$ [36,37], whose optimal frequency is $\sim 0.1 \mathrm{~Hz}$, their improved sensitivity $\left(\Omega_{\mathrm{GW}} \sim\right.$ $10^{-17}-10^{-15}$ ) could constrain excess power with $\mathcal{A} \sim 1$ at a single wave number at a Hubble entry temperature $\sim 10^{5} \mathrm{GeV}$.

In summary, we have presented the second-order evolution equation for tensor modes driven by quadratic scalar terms, and have solved this to obtain the resulting power spectrum of the gravitational waves. By considering density fluctuations with excess power on a given scale, as well as a nearly scale-invariant power spectrum, we have shown how future gravitational wave detectors can place constraints on the primordial density fluctuations at much smaller scales than can be probed by observing the CMB and large-scale structure.

\section{ACKNOWLEDGMENTS}

The authors would like to thank Marco Bruni, Roy Maartens, Karim Malik, and Jean-Philippe Uzan for useful comments and discussions. K. N. A. acknowledges financial support from PPARC. 


\section{APPENDIX A: SECOND-ORDER TENSOR EVOLUTION EQUATION}

In this section we consider the evolution equations for gravitational waves sourced by first-order scalar perturbations. We start with the trace-reversed Einstein field equations (EFE's)

$$
\bar{R}_{\alpha \beta}=\kappa^{2}\left(\bar{T}_{\alpha \beta}-\frac{1}{2} \bar{g}_{\alpha \beta} \bar{T}\right)=\kappa^{2} \bar{S}_{\alpha \beta},
$$

where $\kappa^{2}=8 \pi G$. We consider perturbations up to second order about a Robertson-Walker background. We will consider purely scalar degrees of freedom at first order and tensor degrees of freedom at second order [i.e. we consider a metric of the form Eq. (2) and similar assumptions are made for the energy momentum tensor (EMT)]. Additionally, we choose the longitudinal gauge at first order and assume no anisotropic pressure at first order. We expand the trace-reversed EFE's up to quadratic terms and require the space-space part of the equations at second order. The second-order Ricci tensor may be schematically written as

$$
\delta^{2} R_{\alpha \beta}=\frac{1}{2} \delta^{2} R_{\alpha \beta}^{T}+\delta^{2} R_{\alpha \beta}^{(S, S)} .
$$

The first term $\left(\delta^{2} R_{\alpha \beta}^{T}\right)$ only contains purely second-order terms (i.e. $h_{i j}$ and its derivatives)

$$
\delta^{2} R_{\alpha \beta}^{T}=\frac{1}{4} h_{i j}^{\prime \prime}+\frac{\mathcal{H}}{2} h_{i j}^{\prime}+\frac{1}{2}\left(\frac{a^{\prime \prime}}{a}+\mathcal{H}^{2}\right) h_{i j}-\frac{1}{4} \nabla^{2} h_{i j} .
$$

The remaining term $\left(\delta^{2} R_{\alpha \beta}^{(S, S)}\right)$ represents the extra terms which are quadratic in first-order scalar perturbations and have been explicitly calculated by other authors (e.g. [24]). Using the notation and assumptions outlined in Sec. II the term may be written as

$$
\begin{aligned}
\delta^{2} R_{i j}^{(S, S)}= & {\left[8\left(\frac{a^{\prime \prime}}{a}+\mathcal{H}^{2}\right) \Phi \Phi+16 \mathcal{H} \Phi \Phi^{\prime}+2 \Phi \Phi^{\prime \prime}\right.} \\
& \left.+2 \Phi^{\prime} \Phi^{\prime}+2 \Phi^{\mid k} \Phi_{\mid k}+2 \Phi \nabla^{2} \Phi\right] \gamma_{i j} \\
& +4 \Phi \Phi_{\mid i j}+2 \Phi_{\mid i} \Phi_{\mid j} .
\end{aligned}
$$

The second-order trace-reversed EMT is then

$$
\begin{aligned}
\delta^{2} S_{i j}= & -a^{2} \Phi(\delta \rho-\delta P) \gamma_{i j}+\frac{a^{2}}{4}(\rho-P) h_{i j} \\
& +a^{2}(\rho+P) \delta v_{i} \delta v_{j} .
\end{aligned}
$$

Additionally, we require the following zeroth and firstorder equations

$$
\begin{aligned}
\frac{a^{\prime \prime}}{a} & =-\mathcal{H}^{2}+\frac{\kappa^{2} a^{2}}{2}(\rho-P), \\
\delta v_{i} & =\frac{-2}{a^{2}(\rho-P)}\left(\mathcal{H} \Phi_{\mid i}+\Phi_{\mid i}^{\prime}\right) .
\end{aligned}
$$

Using the above equations and dropping terms with $\gamma_{i j}$ as a coefficient [the $\gamma_{i j}$ terms are eliminated by the tensor operator $\hat{\mathcal{T}}_{i j}{ }^{l m}$ defined in Eq. (12)], we recover Eq. (13) and (14).

\section{APPENDIX B: COEFFICIENT MATRICES IN $\mathcal{P}_{\boldsymbol{h}}$}

The coefficients which appear in Eq. (25) and elsewhere are presented here. First, we define the column matrices

$$
\begin{gathered}
\mathbf{1}=\left(\begin{array}{l}
1 \\
1 \\
1 \\
1
\end{array}\right), \quad \mathbf{0}=\left(\begin{array}{l}
0 \\
0 \\
0 \\
0
\end{array}\right), \quad \boldsymbol{a}=\left(\begin{array}{l}
-1 \\
-1 \\
+1 \\
+1
\end{array}\right), \\
\boldsymbol{b}=\left(\begin{array}{c}
-1 \\
+1 \\
+1 \\
-1
\end{array}\right), \quad \boldsymbol{c}=\left(\begin{array}{c}
+1 \\
-1 \\
+1 \\
-1
\end{array}\right) .
\end{gathered}
$$

Then we may write

$$
\alpha_{n}=\left(\begin{array}{c}
\frac{u}{\sqrt{3}} \mathbf{1}-\frac{v}{\sqrt{3}} \boldsymbol{a}+\boldsymbol{c} \\
\frac{u}{\sqrt{3}} \mathbf{1}-\frac{v}{\sqrt{3}} \boldsymbol{a}+\boldsymbol{c}
\end{array}\right), \quad \phi_{n}=\frac{\pi}{2}\left(\begin{array}{l}
\mathbf{0} \\
\mathbf{1}
\end{array}\right)
$$

and

$$
\begin{aligned}
& M_{n m}^{1}=\left(\begin{array}{ccccc}
\mathbf{0} & \frac{\sqrt{3}}{12} u^{2} v \mathbf{1}-\frac{\sqrt{3}}{36} u v^{2} \boldsymbol{a} & \mathbf{0} & \frac{\sqrt{3}}{2}(u \boldsymbol{a}-\boldsymbol{v} \mathbf{1}) & \mathbf{0} \\
\frac{1}{36} u^{2} \boldsymbol{v}^{2} \boldsymbol{a} & \mathbf{0} & -\frac{1}{12}\left(3 u^{2}+\boldsymbol{v}^{2}\right) \boldsymbol{a}+\frac{1}{2} u v \mathbf{1} & \mathbf{0} & \frac{3}{2} \boldsymbol{a}
\end{array}\right), \\
& M_{n m}^{2}=\left(\begin{array}{ccccc}
\frac{1}{36} u^{2} v^{2} \boldsymbol{b} & \mathbf{0} & -\frac{1}{12}\left(3 u^{2}+v^{2}\right) \boldsymbol{b}+\frac{1}{2} u v \boldsymbol{c} & \mathbf{0} & \frac{3}{2} \boldsymbol{b} \\
\mathbf{0} & -\frac{\sqrt{3}}{12} u^{2} v \boldsymbol{c}-\frac{\sqrt{3}}{36} u v^{2} \boldsymbol{b} & \mathbf{0} & \frac{\sqrt{3}}{2}(-u \boldsymbol{b}+v \boldsymbol{c}) & \mathbf{0}
\end{array}\right), \\
& M_{n m}^{3}=\left(\begin{array}{ccccc}
\mathbf{0} & \frac{\sqrt{3}}{12} u^{2} v \mathbf{1}-\frac{\sqrt{3}}{36} u v^{2} \boldsymbol{a} & 0 & \frac{\sqrt{3}}{2}(u \boldsymbol{a}-\boldsymbol{v} \mathbf{1}) & \mathbf{0} \\
\frac{1}{36} u^{2} \boldsymbol{v}^{2} \boldsymbol{a} & \mathbf{0} & -\frac{1}{6}\left(u^{2}+\boldsymbol{v}^{2}\right) \boldsymbol{a}+\frac{1}{2} u v \mathbf{1} & \mathbf{0} & \frac{3}{2} \boldsymbol{a}
\end{array}\right),
\end{aligned}
$$




$$
M_{n m}^{4}=\left(\begin{array}{ccccc}
\frac{1}{36} u^{2} \boldsymbol{v}^{2} \boldsymbol{b} & \mathbf{0} & -\frac{1}{6}\left(u^{2}+\boldsymbol{v}^{2}\right) \boldsymbol{b}+\frac{1}{2} u v \boldsymbol{c} & \mathbf{0} & \frac{3}{2} \boldsymbol{b} \\
\mathbf{0} & -\frac{\sqrt{3}}{12} u^{2} v \boldsymbol{c}+\frac{\sqrt{3}}{36} u v^{2} \boldsymbol{b} & \mathbf{0} & \frac{\sqrt{3}}{2}(-u \boldsymbol{b}+v \boldsymbol{c}) & \mathbf{0}
\end{array}\right) .
$$

[1] A. A. Starobinskii, Pis'ma Zh. Eksp. Teor. Fiz. 30, 719 (1979).

[2] V. A. Rubakov, M. V. Sazhin, and A. V. Veryaskin, Phys. Lett. 115B, 189 (1982).

[3] R. Fabbri and M. d. Pollock, Phys. Lett. 125B, 445 (1983).

[4] L. F. Abbott and M. B. Wise, Nucl. Phys. B244, 541 (1984).

[5] L. Knox and Y.-S. Song, Phys. Rev. Lett. 89, 011303 (2002).

[6] K. Tomita, Prog. Theor. Phys. 37, 831 (1967).

[7] S. Matarrese, O. Pantano, and D. Saez, Phys. Rev. D 47, 1311 (1993).

[8] S. Matarrese, O. Pantano, and D. Saez, Phys. Rev. Lett. 72, 320 (1994).

[9] S. Matarrese, S. Mollerach, and M. Bruni, Phys. Rev. D 58, 043504 (1998).

[10] H. Noh and J.-c. Hwang, Phys. Rev. D 69, 104011 (2004).

[11] C. Carbone and S. Matarrese, Phys. Rev. D 71, 043508 (2005).

[12] C. Carbone, C. Baccigalupi, and S. Matarrese, Phys. Rev. D 73, 063503 (2006).

[13] K. Nakamura, Phys. Rev. D 74, 101301 (2006).

[14] K. Nakamura, Prog. Theor. Phys. 117, 17 (2007).

[15] D. N. Spergel et al., arXiv:astro-ph/0603449.

[16] S. Mollerach, D. Harari, and S. Matarrese, Phys. Rev. D 69, 063002 (2004).

[17] A. R. Liddle and A. M. Green, Phys. Rep. 307, 125 (1998).

[18] R. Easther and E. A. Lim, J. Cosmol. Astropart. Phys. 04 (2006) 010.

[19] S. Y. Khlebnikov and I. I. Tkachev, Phys. Rev. D 56, 653 (1997).
[20] B. Bassett, Phys. Rev. D 56, 3439 (1997).

[21] G. N. Felder and L. Kofman, Phys. Rev. D 75, 043518 (2007).

[22] http://www.ligo.caltech.edu/.

[23] The Greek indices run from $0-3$ and Latin indices run from $1-3$

[24] V. Acquaviva, N. Bartolo, S. Matarrese, and A. Riotto, Nucl. Phys. B667, 119 (2003).

[25] K. N. Ananda, Ph.D. thesis, University of Portsmouth, 2006.

[26] K. N. Ananda, C. Clarkson, M. Bruni, and D. Wands (to be published).

[27] I. S. Gradshteyn and I. M. Ryzhik, Table of Integrals, Series, and Products (Academic Press, London, 1980), 5th ed.

[28] M. Maggiore, Phys. Rep. 331, 283 (2000).

[29] http://lisa.jpl.nasa.gov/.

[30] N. Seto, S. Kawamura, and T. Nakamura, Phys. Rev. Lett. 87, 221103 (2001).

[31] H. Kudoh, A. Taruya, T. Hiramatsu, and Y. Himemoto, Phys. Rev. D 73, 064006 (2006).

[32] Y. Watanabe and E. Komatsu, Phys. Rev. D 73, 123515 (2006).

[33] http://www.geo600.uni-hannover.de/.

[34] http://tamago.mtk.nao.ac.jp/.

[35] http://wwwcascina.virgo.infn.it/.

[36] V. Corbin and N. J. Cornish, Classical Quantum Gravity 23, 2435 (2006).

[37] G. M. Harry, P. Fritschel, D. A. Shaddock, W. Folkner, and E. S. Phinney, Classical Quantum Gravity 23, 4887 (2006). 